\title{
Why it Remains Difficult for Remote Cardiologist to Obtain the Locus of Control for Ambulatory Health Care Conditions Such as Congestive Heart Failure?
}

\author{
lyngkaran $\mathbf{P}^{1 *}$, Brown $\mathrm{A}^{2}$, Cass $\mathrm{A}^{3}$, Battersby $\mathbf{M}^{4}$, Nadarajan $\mathrm{K}^{5}$ and Ilton $\mathbf{M}^{6}$ \\ ${ }^{1}$ Cardiologist Royal Darwin Hospital, Senior Lecturer Flinders University, Research Fellow FHBHRU, Darwin Private Hospital, Australia \\ ${ }^{2}$ Aboriginal Research Theme Leader, SAHMRI, Australia \\ ${ }^{3}$ Director Menzies School of Health Research, Australia \\ ${ }^{4} \mathrm{Head}$, Flinders Human Behaviour and Health Research Unit (FHBHRU) Margaret Tobin Centre, Flinders University, Australia \\ ${ }^{5}$ Cardiologist and Co-Director Division of Medicine, Royal Darwin Hospital, Australia \\ ${ }^{6}$ Director of Cardiology, Royal Darwin Hospital, Australia
}

"...it is essential that those held accountable have the processes of care being assessed under their locus of control...”

\section{Harlan Krumholz}

Clinicians and researchers aiming to improve congestive heart failure $(\mathrm{CHF})$ services need to invest in quality assurance research. In this regard standards of accountability can be met for the goals set within any particular programme. Part of this process involves gathering evidence, which is the basis for audit and observational research. Implementing these findings can be straight forward when the issues are limited to resources. In all cases the responses can be negative, positive or somewhat positive where certain measures are taken within the constraints of that system. These issues becomes more difficult when findings involve implementing a new strategy or if there are more than one choice available. We have previously discussed some of these challenges and potential solutions for the NT [1-5]. In this short commentary, we discuss briefly some issues on the implementation strategies for CHF best practice in the Northern Territory (NT) of Australia and why it remains difficult for the cardiologists who are held to account to also maintain a degree of control on the process.

The position statement on performance measures complemented with the above statement was designed to encourage clinician led research practice [6,7]. The advantage of this approach is that it encourages those who are providing a service, being assessed for that services, a consensus derived platform to maintain a degree of control over this process. In fact, this statement may have greater significance in remote and areas of need where local knowledge could play a part in best practice. This statement was probably, not intended to encourage unilateral decisions or a hierarchical approach from any one group. However, despite encouraging clinicians to obtain a degree of control, without collaborative approaches, the process may appear otherwise.

In the acute setting regardless of environment the locus of control is often within the cardiologist grasp as most decision are made at the bedside. The grey areas start at discharge and extend further with ambulatory conditions. CHF can be largely managed in the community with more intensive specialist care during periods of decompensation. In the NT hospital cardiology focused acute care is only possible in one hospital, the Royal Darwin Hospital, while cardiology supervised subacute care is only possible in Darwin. In the NT the locus of control for a significant number of acute care cases and greater number of subacute cases are not within cardiology control. In stable cases, while the urgency of specialty consultation is less there are still challenges. In this regard we have set up early measures to collect prospective data [4] and pilot supporting intervention tools $[5]^{\mathrm{a}}$.

In the end obtaining data alone may not be sufficient. Importantly, clinicians should explore strategies to implement these research findings. To do so the evidence gathered needs to be robust, durable, explore measures of cost economics wide reaching and remain externally valid for a broad demographic. We have to be convincing in our findings to influence other prescribers (general practitioners, other subspecialists, nurse practitioners) and policy makers who can determine the success of HF management programmes. Additional challenges that necessitate research strategies with broad aims are the funding and staffing availability, or relative scarcity. In a sense research strategies need to be multidimensional with creative use of funding, where staff also multi-task, which could have consequences for the depth of information and validity of findings. Unfortunately efficiency in the research sense is synonymous in economic terms due to issues of validity.

Collecting research data in the NT has become relatively easier. Research infrastructure has been strengthened and assistance from partners has helped. Although there are these positive developmental elements, we are keen to highlight some areas of contention e.g. the performance measures added and/or rested in the NTHFI-CA (4). The questions we anticipate when the results are available: Firstly, will the rationale for the decisions made be free from scrutiny, i.e. without significant precedence from trials in the region significant assumptions have to me made; and secondly, the application of results to a larger audience outside the NT i.e will federal policy makers be persuaded about the cost effectiveness of strategies for this populations. This first hurdle usually does not greatly affect strategies that call for increased resources in staffing and infrastructure. As we have previously highlighted this one-dimensional strategy may not however in the long term achieve the programme goals $(1,2)$. Thus in our case, should

aNorthern Territory Heart Failure Initiative - 2 Worlds Study (NTHFI -2Worlds) - a third study in progress, aimed at assessing an Indigenous patient journey mapping tool in a prospective pilot. Study details are in preparation and will be published when completed.

*Corresponding author: $\mathrm{Dr}$ Pupalan lyngkaran Cardiologist Royal Darwin Hospital, Senior Lecturer Flinders University, Research Fellow FHBHRU, Darwin Private Hospital, Rocklands Drive, Tiwi, NT 0811, Australia, Tel +618 84042323 E-mail: balaniyngkaran@hotmail.com

Received January 07, 2014; Accepted January 20, 2014; Published January 25, 2014

Citation: lyngkaran P, Brown A, Cass A, Battersby M, Nadarajan K, et al. (2014) Why it Remains Difficult for Remote Cardiologist to Obtain the Locus of Control for Ambulatory Health Care Conditions Such as Congestive Heart Failure? A Tug of War between General Practice, Administrators and Implementable Research Findings. J Gen Pract 2: 146. doi: 10.4172/2329-9126.1000146

Copyright: (c) 2014 lyngkaran P, et al. This is an open-access article distributed under the terms of the Creative Commons Attribution License, which permits unrestricted use, distribution, and reproduction in any medium, provided the original author and source are credited. 
Citation: lyngkaran P, Brown A, Cass A, Battersby M, Nadarajan K, et al. (2014) Why it Remains Difficult for Remote Cardiologist to Obtain the Locus of Control for Ambulatory Health Care Conditions Such as Congestive Heart Failure? A Tug of War between General Practice, Administrators and Implementable Research Findings. J Gen Pract 2: 146. doi: 10.4172/2329-9126.1000146

Page 2 of 4

$\checkmark$ WHAT HAVE WE ACHIEVED?

$\checkmark$ WHAT ARE THE MANOR STUMBLING BLOCKS?

$\checkmark$ WHAT ARE THE FUNDING AND ADMINISTRATIVE ISSUES?

$\checkmark$ WHAT ARE THE DIFFICULTIES IN ENGAGING WITH PRIMARY CARE?

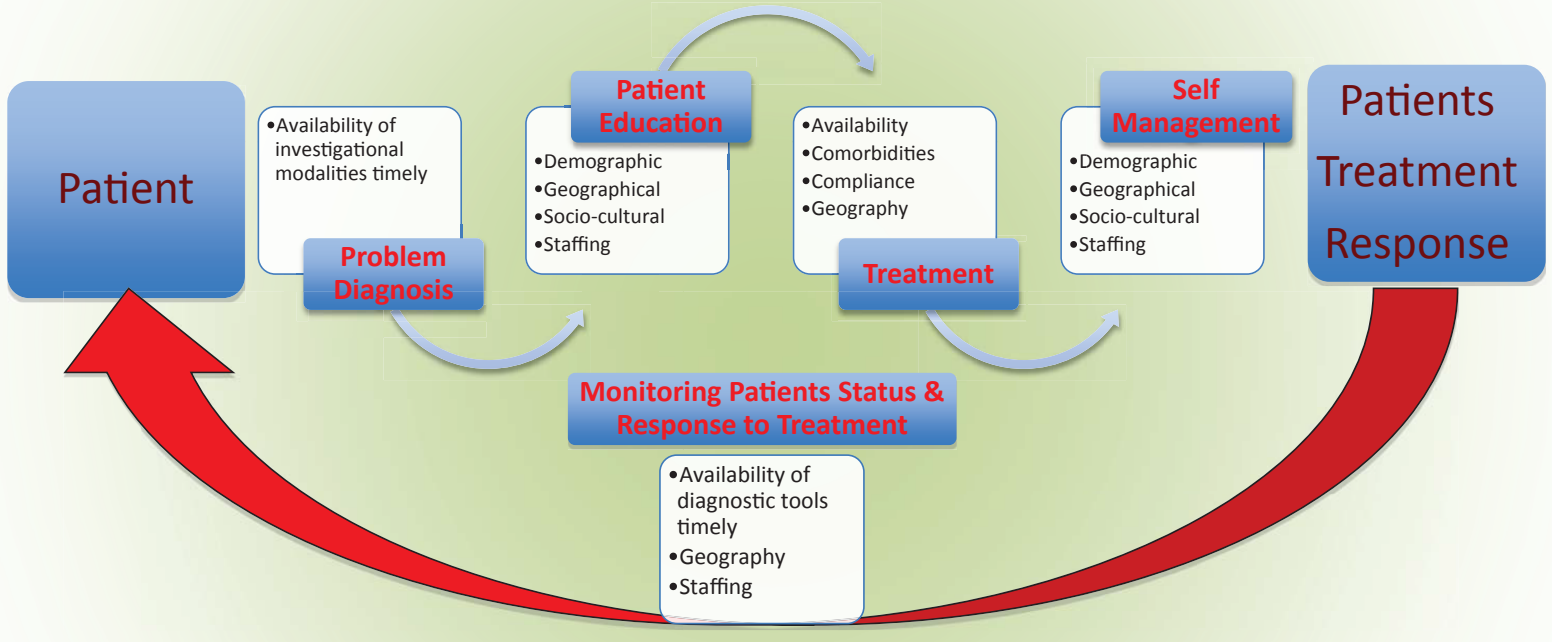

$\mathrm{CHF}$ treatment pathways require patient interaction at primary and tertiary levels with general practitioners, specialists and allied healt staff. Challenges for best practice in the domains range from demographic, geographical, infrastructure, socio-cultural and staffing factors. Gathering information and providing solutions for each dimension of care will require acknowledging achievements, exploring stumbling blocks and creating new strategies. Such approaches to be successful require collaborative, efficient and innovative strategies. This is likely to provide confidence from all sides in implementing the strategies and secure ongoing funding. It is likely that the locus of control for ambulatory health conditions such as $\mathrm{CHF}$ in remote regions lies with the systems. It is however still important that cardiologist be the drivers, and/or leaders for these specialty conditions across the spectrum of care. (Image modified from Ref 6 )

Figure1: Congestive Heart Failure Treatment Pathway and Barriers.

any generated data support exploring interventions beyond staffing or infrastructure funding, it remains to be seen how this will affect thinking for intervention strategies particularly in therapeutics, chronic disease self-management programmes (CDSMP) or technology assisted programmes, to name a few, should they be required. More than likely in moving forward we will require further research in multifaceted intervention studies. It is fortunate in Australia the NHMRC ${ }^{\mathrm{b}}$ does encourage such efforts and a more likely to support collaborative efforts between clinicians, research institutes and local health department who provide in kind funding for the interventional study.

Structuring interventional research studies is not a difficult process when funding has been secured. The gold standard RCT may unfortunately not be the most suitable study to conduct. While maintaining a strong internal validity two issues are worth highlighting. Firstly, the NT population is vulnerable and a placebo arm may not receive ethics committee support. An example here was the SHARP study on Vytorin, where there was concern of the effects of placebo on an already at risk group. Secondly, are the issues of the comparator. Non-inferiority studies would be supported, this will however require a baseline study to compare and agreement on the comparator. This may be less contentious for hospital based therapeutics, but more so

${ }^{\mathrm{b} N a t i o n a l}$ Health and Medical Research Council - Collaboration and Programme Grants

${ }^{\mathrm{C}}$ The Flinders Program ${ }^{\mathrm{TM}}$ for Chronic Condition Management: Information Paper http://som.flinders.edu.au/FUSA/CCTU/Home.html for community based interventions. An example we highlight here has been in shaping a CDSMP, as part of a CHF disease management programme. The locally generated and federal government supported CFPI $^{c}$, has had some stumbling blocks in the NT due to concerns of time and user friendliness and application to a broad clientele e.g. Indigenous Australians. Thus any study on CDSMP will require trialing several strategies some favored by administrators, others by clinicians and/or allied health, who implements these strategies. Furthermore there is a need to convince general practitioners, who appear to be the key for CDSMP success $[8,9]$, to participate, while there remain ongoing concerns on remuneration, practice staffing to complete and follow-up along with individual practice preferences. Finally, while some may argue that community based strategies are not within the domain of cardiologist, it may be that in remote strategies the distinction and boundaries between primary care and tertiary care will actually become less defined purely from the sheer numbers of patients, the illness burden with comorbidities and staffing shortfalls. In this case community strategies for ambulatory conditions could receive some primary care at tertiary centers and followed through remotely and vice-versa, as a measure in efficiency. A consensus on the approach and sharing of information will be part of this new paradigm.

The ideal interventional research study for this region would have the primary aims of patient and staff self-reported satisfaction (or technology uptake) and health economics, with secondary aims of outcomes and within a quasi-experimental design. This will address issues of compliance, staff user friendliness and whether the system 
Citation: Iyngkaran P, Brown A, Cass A, Battersby M, Nadarajan K, et al. (2014) Why it Remains Difficult for Remote Cardiologist to Obtain the Locus of Control for Ambulatory Health Care Conditions Such as Congestive Heart Failure? A Tug of War between General Practice, Administrators and Implementable Research Findings. J Gen Pract 2: 146. doi: 10.4172/2329-9126.1000146

will invest in this approach for the long term. While outcomes are the primary aims of many RCT, we have to accept that merely implementing RCT findings has not generated the desired clinical outcomes. Of concern, is the limited emphasis on phase 4 post marketing data to further explore the outcomes of new therapies in a regional setting. Perhaps, it could be argued that policy makers do assume that the findings from RCT will generate the necessary benefits as advertised. It is thus important that we explore step lock measures in research; for e.g. as highlighted many systems readily implement findings from RCT when questions of external validity still remain. In this should we not assume that if we implement a strategy that improves compliance and delivery i.e meeting the RCT design, would these also not equate to outcomes, thus negating the need for outcome measures in this study. Alternatively exploring quasi-experimental trial designs. Benefits of this approach is that the results are likely to have high external validity, allow for a broad experimental intervention and replicate real world clinical conditions [9-11]. To date there have been no such trials on a large scale for CHF, nor has any such trial significantly influenced policy for any cardiovascular condition, to our knowledge. Asch et al. however using this design was able to demonstrate the benefits of a collaborative organizational care intervention in CHF [12]. Other small but limited examples for CHF have also been published [12-15]. For cardiovascular disease as a whole, some large studies have been reported, mostly positive [6-23], although longer term translation of findings into actual clinical care within those systems is unknown. Failures have also been seen with this approach in the British NHS [24]. Findings from other studies are also awaited [25]. It remains to be seen that if we choose to go down this pathway when more data becomes available $[4,5]$ would the funding bodies and policy makers equally support collaborative programme funding with this design.

In summary, in remote care there are alternative prescribers for therapy, policy makers who decide on deliverable services and a difficult demographic to conduct research studies where the findings could influence policy. This would appear to create a tug-of-war for control of CHF care away from the cardiologist. This would appear to create a tug-of-war for control of CHF care away from the cardiologist. Optimizing $\mathrm{CHF}$ care in this setting remains difficult, but not from lack of intent. While cardiology control over the process is variable, accountability is still required. In moving forward, for cardiologist to assume greater control, we could support the need for cardiologist driven efforts to increase their credibility by steering the regional evidence generating processes. This process requires acknowledging the existing paradigm and tailoring studies to generate credible evidence to support the newer paradigms. In gathering this evidence we propose several points that are worth considering: Firstly, there are situations where local interpretation of evidence matched with experimental trial designs are probably required. Whether this is a good way forward is uncertain however it allows for us to maximize the information we gain and explore implementing broad collaborative strategies; Secondly, exploring alternate interventional studies apart from the gold standard RCT to broaden intervention options with a wide exernal appeal. This may remain until there are further efforts to define:

- Principles on interpreting external validity of studies [3]

- Importance of physiological modulation behind the drug therapies, thus broadening the acceptability for physiological based prescribing principles for future studies in CHF, particularly with comorbidities [2,3]

- The axis of control for subspecialty ambulatory health care conditions that require collaborative and multifaceted approaches to research practice [1]
It would appear to improve CHF care; there is a need for a broader range of interventional research tools that focus on implementation in a heterogeneous group and allow a broader range of questions to be answered, that can similarly steer policy. We would encourage clinicians in similar clinical settings to contribute further in this area. Thus the locus of control for CHF in remote practice lies within the system broadly. It is important however that cardiologist, who are the principal prescribers, also accept the greatest share of responsibility for outcomes. In doing so cardiologist, should take a leading role in steering the discussion and ensuring accountability with a colleagueble spirit and collaborative approach for this sub-specialty ambulatory care condition (Figure 1).

\section{Disclosures}

All co-authors have won independent and governmental research funding. None pose a conflict of interest for this paper. Dr Iyngkaran is supported by the RACP Fellow Contribution research award.

\section{References}

1. lyngkaran P, Harris M, Nadarajan K, Ilton M, Battersby M, et al. (2014) Implementing Guideline Based Heart Failure Care in the Northern Territory: Challenges and Solutions. Heart Lung \& Circ Feb 2014.

2. lyngkaran P, Thomas M, Sanders P, Hare DL, Majoni W, et al. (2013) Do we need a wider therapeutic paradigm for heart failure with comorbidities? A remote Australian Perspective. Health Care Current Reviews 1: 106.

3. lyngkaran P, Thomas M, Majoni W, Anavekar NS, Ronco C (2012) Comorbid Heart Failure and Renal Impairment: Epidemiology and Management Cardiorenal Med 2: 281-297.

4. Iyngkaran P, Tinsley J, Smith D, Haste M, Nadarajan K, et al. (2014) Northern Territory Heart Failure Initiative Clinical Audit (NTHFI - CA) A Prospective Database on the Quality of Care and Outcomes for Acute Decompensated Heart Failure Admission in the Northern Territory - Study Design and Rationale. BMJ Open 2014:4:e004137.

5. lyngkaran P, Majoni V, Nadarajan K, Haste M, Battersby M, et al. (2013) AUStralian Indigenous Chronic Disease Optimisation Study (AUSI-CDS) prospective observational cohort study to determine if an established chronic disease health care model can be used to deliver better heart failure care among remote Indigenous Australians: Proof of concept-study rationale and protocol. Heart Lung Circ 22: 930-939.

6. Spertus JA, Bonow RO, Chan P, Diamond GA, Drozda JP Jr, et al (2010) ACCF/AHA new insights into the methodology of performance measurement a report of the American College of Cardiology Foundation/American Heart Association Task Force on performance measures. Circulation 122: 2091-2106.

7. Krumholz HM, Currie PM, Riegel B, Phillips CO, Peterson ED, et al. (2006) A taxonomy for disease management: a scientific statement from the American Heart Association Disease Management Taxonomy Writing Group. Circulation 114: $1432-1445$.

8. Jordan JE, Osborne RH (2007) Chronic disease self-management education programs: challenges ahead. Med J Aust 186: 84-87.

9. Ho PM, Peterson PN, Masoudi FA (2008) Evaluating the evidence: is there a rigid hierarchy? Circulation 118: 1675-1684.

10. Harris AD, McGregor JC, Perencevich EN, Furuno JP, Zhu J, et al. (2006) The use and interpretation of quasi-experimental studies in medical informatics. $J$ Am Med Inform Assoc 13: 16-23.

11. Linden A, Adams JL (2008) Improving participant selection in disease management programmes: insights gained from propensity score stratification. J Eval Clin Pract 14: 914-918

12. Asch SM, Baker DW, Keesey JW, Broder M, Schonlau M, et al. (2005) Does the collaborative model improve care for chronic heart failure? Med Care 43 . 667-675.

13. Lowery J, Hopp F, Subramanian U, Wiitala W, Welsh DE, et al. (2012) Evaluation of a nurse practitioner disease management model for chronic heart failure: a multi-site implementation study. Congest Heart Fail 18: 64-71. 
Citation: lyngkaran P, Brown A, Cass A, Battersby M, Nadarajan K, et al. (2014) Why it Remains Difficult for Remote Cardiologist to Obtain the Locus of Control for Ambulatory Health Care Conditions Such as Congestive Heart Failure? A Tug of War between General Practice, Administrators and Implementable Research Findings. J Gen Pract 2: 146. doi: 10.4172/2329-9126.1000146

14. Dykes PC, Acevedo K, Boldrighini J, Boucher C, Frumento K, et al. (2005) Clinical Practice Guideline Adherence Before and After Implementation of the HEARTFELT (HEART Failure Effectiveness \& Leadership Team) Intervention Journal of Cardiovascular Nursing 20(5): 306-314.

15. Wang SP, Lin LC, Lee CM, Wu SC (2011) Effectiveness of a self-care program in improving symptom distress and quality of life in congestive heart failure patients: a preliminary study. J Nurs Res 19: 257-266.

16. Unruh MA, Trivedi AN, Grabowski DC, Mor V (2013) Does reducing length of stay increase rehospitalization of medicare fee-for-service beneficiaries discharged to skilled nursing facilities? J Am Geriatr Soc 61: 1443-1448.

17. McWilliams JM, Meara E, Zaslavsky AM, Ayanian JZ (2009) Differences in control of cardiovascular disease and diabetes by race, ethnicity, and education: U.S. trends from 1999 to 2006 and effects of medicare coverage. Ann Intern Med 150: 505-515.

18. Ogilvie D, Griffin S, Jones A, Mackett R, Guell C, et al. (2010) Commuting and health in Cambridge: a study of a 'natural experiment' in the provision of new transport infrastructure. BMC Public Health 10: 703.

19. Rabiei K, Kelishadi R, Sarrafzadegan N, Abedi HA, Alavi M, et al. (2009) Process evaluation of a community-based program for prevention and contro of non-communicable disease in a developing country: The Isfahan Healthy Heart Program, Iran. BMC Public Health 9: 57.

20. Li P, McElligott S, Bergquist H, Schwartz JS, Doshi JA (2012) Effect of the Medicare Part $D$ coverage gap on medication use among patients with hypertension and hyperlipidemia. Ann Intern Med 156: 776-784.
21. Vallès-Fernandez R, Rosell-Murphy M, Correcher-Aventin O, Mengual-Martínez L, Aznar-Martínez N, et al. (2009) A quality improvement plan for hypertension control: the INCOTECA Project (INterventions for COntrol of hyperTEnsion in CAtalonia). BMC Public Health 9: 89.

22. Nguyen QN, Pham ST, Nguyen VL, Weinehall L, Wall S, et al. (2012) Effectiveness of community-based comprehensive healthy lifestyle promotion on cardiovascular disease risk factors in a rural Vietnamese population: a quasi-experimental study. BMC Cardiovasc Disord 12: 56.

23. Dalmau R, Boira M, Aguilar C, López C, Rodríguez D, et al. (2011) Lipidlowering drugs in ischaemic heart disease: a quasi-experimental uncontrolled before-and-after study of the effectiveness of clinical practice guidelines. BMC Cardiovasc Disord 11: 47.

24. Tudor-Smith C, Nutbeam D, Moore L, Catford J (1998) Effects of the Heartbeat Wales programme over five years on behavioural risks for cardiovascular disease: quasi-experimental comparison of results from Wales and a matched reference area. BMJ 316: 818-822.

25. Tricco AC, Soobiah C, Antony J, Hemmelgarn B, Moher D, et al. (2013) Interventions to decrease the risk of adverse cardiac events for post-surgery or chemotherapy patients taking serotonin (5-HT3) receptor antagonists: protocol for a systematic review and network meta-analysis. Syst Rev 2: 45 . 